\title{
APPLICATION OF PARALLEL DISTRIBUTED LAGRANGE MULTIPLIER TECHNIQUE TO SIMULATE COUPLED FLUID-GRANULAR FLOWS IN PIPES WITH VARYING CROSS-SECTIONAL AREA
}

\author{
Yuliya Kanarska ${ }^{1}$, Otis Walton \\ Lawrence Livermore National Laboratory \\ ee-mail: kanarska1@1lnl.gov
}

Keywords: fluid-granular flows, pneumatic conveying, Lagrange multiplier

\begin{abstract}
Fluid-granular flows are common phenomena in nature and industry. Here, an efficient computational technique based on the distributed Lagrange multiplier method is utilized to simulate complex fluid-granular flows. Each particle is explicitly resolved on an Eulerian grid as a separate domain, using solid volume fractions. The fluid equations are solved through the entire computational domain, however, Lagrange multiplier constrains are applied inside the particle domain such that the fluid within any volume associated with a solid particle moves as an incompressible rigid body. The particle-particle interactions are implemented using explicit force-displacement interactions for frictional inelastic particles similar to the DEM method (Cundall and Strack, 1979) with some modifications using the volume of an overlapping region as an input to the contact forces. A parallel implementation of the method is based on the SAMRAI (Structured Adaptive Mesh Refinement Application Infrastructure) library.

Application of this method to simulate fluid-granular flows in pipes with a step decrease in cross-sectional area is discussed. Correlations between pressure losses and solid mass fluxes in a pipe for different particle concentrations, pipe diameters and particle sizes are studied. Results of the simulations agree well with available experimental data for the mass flow ratio vs pressure drop during conveying.
\end{abstract}

\section{INTRODUCTION}

Natural and manmade particulate flows are common in a wide range of industrial applications and in nature. An important application of fluid-granular flows is pneumatic conveying. It is a common method for the transporting granular media, where grains are driven through pipes by air flow and it is widely used in the food industry and in industrial, pharmaceutical and chemical engineering. Despite extensive experimental and theoretical research in this area (see overview in Marcus et al., 2010), prediction of solids transport through specific conveying system designs is still a very challenging problem. Decreasing the energy loss through the conveying system is very critical for increasing its performance. The fundamental 
physics of such flows dominated by two-way coupled fluid/particle interactions is still poorly understood. Therefore development of reliable computational tools and experiments for more precise understanding of the fluid-solid interaction during conveying is essential for better prediction of the transport properties of solids in conveying systems.

The motion of particles is usually classified in two regimes: dilute regime, where the grains move as a suspension in the conveying air, and dense regime, where the grains are subject to long lasting collisions and move in bulk along the pipe. In this study we will consider the air and particle flow in the first regime that is valid for low concentrations of particles and high conveying air velocities. Usually it is considered that the pressure and energy losses for such flows in a pipe with constant cross-sectional area are mainly arising from the frictional and drag forces in a steady state. Much less attention is paid to the energy losses due to flow and particle acceleration effects due to sudden expansions or constrictions that contribute to the overall pressure losses in a pipe. Several analytical models that account for the flow acceleration with air flow (Martin and Pabbi, 1960; Kojasoy et al., 1997) and particulate flow (Rhodes, 2001; Gundogdu et al., 2009) were derived.

In this paper we generalize analytical findings and complement them by numerical results and experimental data. In order to analyze and quantify such fluid-granular flows, we perform direct numerical simulations using the distributed Lagrange multiplier technique (Glowinski et al., 1999, Patankar, 2001, Kanarska et al., 2011) and compare them with experiment conducted at Lawrence Livermore National Laboratory. A systematic variation of the flow and particle parameters is part of an on-going simulation study of fluid-solid granular flows through relatively small pipes. These results will help to predict energy and momentum losses during pneumatic conveying in the systems with variable cross-sectional area. We present step-by-step existing theoretical approaches to predict pressure losses in the system, starting with the simple air flow (without particles) in section 2.1 and section 2.2. And then, similar to the theoretical expressions found for air flows we derive expressions to predict pressure drop for particulate flows in section 2.3 and 2.4. The numerical method is described in section 3 and numerical results are presented in section 4. Comparisons with experimental data are discussed in section 5. Section 6 contains summary and conclusions of the present study.

\section{THEORETICAL APPROACHES}

\subsection{Momentum balance equation in the pipe with air flow}

We consider first incompressible fluid flow (without particles) through a pipe with varying cross-sectional area (Figure 1). We define instant gas velocity as $V_{g}$, pressure $P$ and gas-wall friction force as $F_{g w}$. One may write momentum equations for X-components (where the Xaxis corresponds with the pipe axis) as:

$$
\rho_{g}\left(V_{g x} \frac{\partial V_{g x}}{\partial x}+V_{g y} \frac{\partial V_{g x}}{\partial y}+V_{g z} \frac{\partial V_{g x}}{\partial z}\right)=-\frac{\partial P}{\partial x}+F_{g w}
$$


We integrate this equation across a perpendicular slice $\mathrm{YZ}$ and assume that the flow is symmetric relative to the $\mathrm{X}$-axis. With this assumption equation (1) can be written as

$$
\rho_{g} u_{g x} \frac{\partial u_{g x}}{\partial x}=-\frac{\partial p}{\partial x}+f_{g w}
$$

In this equation $u_{g x}, f_{g w}, \quad p$ are mean values of velocity, frictional forces and pressure in the YZ slice. Then we can derive an expression for the pressure force acting on the fluid as

$$
\frac{\partial p}{\partial x}=-\rho_{g} u_{g x} \frac{\partial u_{g x}}{\partial x}+f_{g w}
$$

Finally we can find the pressure difference at each point along the $\mathrm{X}$-axis. At this point we neglect the frictional forces and separate the pressure drop due to momentum changes as

$$
p_{0}-p_{x}=\int_{0}^{x}\left[\rho_{g} u_{g x} \frac{\partial u_{g x}}{\partial x}\right] d x=\left.\frac{\rho_{g} u_{g x}^{2}}{2}\right|_{x}-\left.\frac{\rho_{g} u_{g x}^{2}}{2}\right|_{0}
$$

This equation corresponds to well-known Bernoulli's equation for fluid flow in pipes.

\subsection{Energy balance equation for the pipe with air flow}

The fluid flow in the pipe shown in figure 1 can be divided into three areas. Area 1 is taken upstream of the orifice. The flow is characterized by the fluid velocity $u_{1 f}$, and crosssectional area $A_{1}$ and pressure $P_{1}$. Area 2 is inside the orifice opening. All quantities are variable in this area. Area 3 is taken downstream inside the small pipe. Flow properties in this region are similar to region (1) with quantities $P_{3}, u_{3 f}, A_{3}$.

The energy balance equation for the fluid between zones 1 and 3 can be written as:

$$
\frac{u_{1 f}^{2}}{2}+\frac{P_{1}}{\rho}=\frac{u_{3 f}^{2}}{2}+\frac{P_{3}}{\rho}
$$

Mass conservation for both fluid and solid gives expression

$$
m_{1 f}=m_{2 f}=m_{3 f}=m_{f}
$$

where $m_{f}=A u_{f} \rho_{f}$.

From equations (5) and (6) we derive 


$$
P_{1}-P_{3}=0.5 \frac{m_{f}^{2}}{A_{3}^{2} \rho_{f}}-0.5 \frac{m_{f}^{2}}{A_{1}^{2} \rho_{f}}=0.5 \frac{m_{f}^{2}}{A_{3}^{2} \rho_{f}}\left(1-\frac{A_{3}^{2}}{A_{1}^{2}}\right)
$$

This equation is valid if there are no energy losses, e.g. turbulent energy losses or wall frictional losses. Usually such additional losses are included by introducing the discharge coefficient $K_{a}$. Let's assume that the pressure drop due to air in the pipe without particles is equal to $\Delta P_{a i r}$. Then equation (7) can be written as

$$
\Delta P_{a i r}=P_{1}-P_{3}=K_{a} \frac{m_{f}^{2}}{A_{3}^{2} \rho_{f}}
$$

In the general case the discharge coefficient $K_{a}$ depends on the orifice/exit pipe area ratio (or expanding ratio), turbulent losses etc. Sometimes this expression is written as

$$
m_{f}=C_{d} A_{3} \sqrt{2 \rho_{f} \Delta P_{\text {air }}}
$$

The values of $C_{d}=\sqrt{1 / 2 \mathrm{~K}_{\mathrm{a}}}$ for sharp and smooth entrances were calibrated in experiments and can be found, for example, in Martin and Pabbi, (1960). It should be noted that in the case of the air flow without particles the mass balance equation (4) corresponds to the energy equation (8). Therefore the energy and mass balance equations provide the same expressions for the pressure losses in this simple case.

\subsection{Momentum balance equation in the pipe with air flow and particles}

Similar to air flow considered in section 2.1 now we study air flow with particles and derive momentum equations for $\mathrm{X}$-components of velocity for both the gaseous and solid phases as:

$$
\begin{aligned}
& \varepsilon \rho_{g}\left(V_{g x} \frac{\partial V_{g x}}{\partial x}+V_{g y} \frac{\partial V_{g x}}{\partial y}+V_{g z} \frac{\partial V_{g x}}{\partial z}\right)=-\frac{\partial P}{\partial x}+F_{g s}+F_{g w} \\
& (1-\varepsilon) \rho_{s}\left(V_{s x} \frac{\partial V_{s x}}{\partial x}+V_{s y} \frac{\partial V_{s x}}{\partial y}+V_{s z} \frac{\partial V_{s x}}{\partial z}\right)=-F_{g s}+F_{s w}+F_{s c}
\end{aligned}
$$

where $\varepsilon$ is the volume fraction of air which can vary with position in the flow, and $(1-\varepsilon)$ is the volume fraction of solid particles, $V_{s}$ is the solid velocity, $F_{g w}$ and $F_{s w}$ are air-wall and solid-wall friction forces correspondingly, $F_{g s}$ is the interaction force between solids and air and $F_{s c}$ is the particle collisional losses term. By integrating these equations across a perpendicular slice $\mathrm{YZ}$ and assuming that the flow is symmetric relative to the $\mathrm{X}$-axis we derive: 


$$
\begin{aligned}
& \varepsilon \rho_{g} u_{g x} \frac{\partial u_{g x}}{\partial x}=-\frac{\partial p}{\partial x}+f_{g s}+f_{g w} \\
& (1-\varepsilon) \rho_{s} u_{s x} \frac{\partial u_{s x}}{\partial x}=-f_{g s}+f_{s w}+f_{s c}
\end{aligned}
$$

here $u_{g x}, u_{s x}, f_{g s}, f_{g w}, f_{s w}, f_{s c}, p, \varepsilon$ are mean values of corresponding velocity, interaction force between phases, frictional forces, the particle collisional losses term, pressure and volume fraction in the $\mathrm{YZ}$ slice.

Eliminating internal interaction force $f_{g s}$ between phases from equations (12) and (13) yields the following equation for the pressure gradient force acting on the fluid as

$$
\frac{\partial p}{\partial x}=-\varepsilon \rho_{g} u_{g x} \frac{\partial u_{g x}}{\partial x}-(1-\varepsilon) \rho_{s} u_{s x} \frac{\partial u_{s x}}{\partial x}+f_{g w}+f_{s w}+f_{s c}
$$

Integrating equation (14) along the $\mathrm{X}$-axis and neglecting the frictional forces $f_{g w}, f_{s w}$ and $f_{s c}$ in (14) gives the following equation

$$
p_{0}-p_{x}=\int_{0}^{x}\left[\varepsilon \rho_{g} u_{g x} \frac{\partial u_{g x}}{\partial x}+(1-\varepsilon) \rho_{s} u_{s x} \frac{\partial u_{s x}}{\partial x}\right] d x
$$

This equation can be significantly simplified if the solid concentration is constant along the Xaxis. For example, Rhodes (2001) and Gundogdu et al. (2009) derived expressions for the total pressure drop along the pipe assuming constant solid void fraction along the pipe. However, as already mentioned, the particles tend to accelerate while moving through the system. Since the solid mass flux is constant in a steady state flow, the particle concentration decreases along the X-direction. Therefore, strictly speaking, equation (15) needs to be integrated in each cross-section through the pipe. But it still can be used for diagnostic purposes to estimate contributions of the acceleration terms inside the system to the total energy losses. Such an analysis will be presented in section 4 .

\subsection{Generalization of functional forms for the pressure drop with gas flow and particles}

Since equation (15) has an integral form it can be used for numerical diagnostic purposes only. In this section we derive an expression for the pressure drop in more general form. We assume that the global pressure drop is equal to the sum of the air and solid pressure drops as

$$
\Delta P=\Delta P_{\text {air }}+\Delta P_{s}
$$


where the solid pressure $\Delta P_{s}$ describes contribution to the pressure drop in the air due to the presence of particles in the system. This term can be computed similar to the air pressure drop (eq. (16)) by introducing a discharge coefficient for solids $K_{s}$ as

$$
\Delta P-\Delta P_{\text {air }}=\Delta P_{s}=K_{s} \frac{m_{s}^{2}}{A_{3}^{2}(1-\varepsilon) \rho_{s}}
$$

Using equation (8) we can rewrite equation (17) as

$$
\frac{\Delta P-\Delta P_{\text {air }}}{\Delta P_{\text {air }}}==\frac{K_{s}}{K_{a}} \frac{m_{s}}{m_{f}} \frac{u_{s}}{u_{f}}=k_{s} \frac{m_{s}}{m_{f}} \frac{u_{s}}{u_{f}}
$$

An introduced functional form provides correlation between the pressure drop in the system and the solid mass fluxes. For single-phase air flow the discharge coefficient $K_{a}$ is usually defined as a function of the expansion ratio. In the case of two-phase flow of air with particles the flow is more complex and the coefficient $K_{s}$ needs to be defined from numerical simulations and/or experimental data for the given system. If the pipe is sufficiently long and the particles are small (<40 microns) the particles reach fluid velocity at the pipe exit and $\frac{u_{s}}{u_{f}}=1$ in (18). Otherwise if the pipe is relatively short and particles are large the ratio $\frac{u_{s}}{u_{f}}$ needs to be estimated. Application of numerical methods and experimental data to this problem will be discussed in section 4 and 5 .

\section{NUMERICAL METHOD}

While the analytical models above include only momentum changes and ignore unsteady behavior such as flow separation, turbulence and particle collision effects, all of those effects are included in the numerical simulations. The only physical process not included in the simulations is any effects due to the gas density changing with pressure (i.e. the air is incompressible).

The method we used is based on the Distributed Lagrange Multiplier (DLM) technique (Kanarska et al., 2011) that was originally developed to study particulate flows by Glowinski et al., (1999) and Patankar et al., (2001). Each particle is treated as an individual grain and it is explicitly resolved on the Eulerian grid using solid volume fractions. The grain cell size in our method is smaller than the particle diameter (see [6]). The fluid equations are solved throughout the entire computational domain; however, Lagrange multiplier constraints are applied inside the particle domain, such that the fluid within any volume associated with a solid particle moves as an incompressible rigid body. The particles interact with the 
surrounding fluid via fluid dynamics equations, resulting in implicit fluid-rigid-body coupling relations that produce realistic fluid flow around the particles (i.e., no-slip boundary conditions). In addition, particle-particle interactions are implemented using the DEM method (Cundall and Strack, 1979) with frictional, inelastic contact forces. Since the particle domain is explicitly resolved on the Eulerian grid, we slightly modify this method using the overlap volume of contacting particles as the input to the contact forces. The code is parallelized using the Structured Adaptive Mesh Refinement Application Infrastructure (SAMRAI) framework developed at Lawrence Livermore National Laboratory (LLNL) (Hornung and Kohn, 2002). This framework allows tracking individual particle position on multiple central processing units (CPUs) and refining the resolution on a structured grid in areas of interest (e.g., solidfluid interfaces, maximum velocity zones, etc.). Griffith et al. (2007, 2010) extended the SAMRAI infrastructure for fluid-structure interaction problems using an immersed boundary method (Peskin, 1977). Our approach extends this framework using the DLM technique to describe fluid-solid interactions for the case of a large number of rigid particles. More detailed description of the method along with the governing equations and algorithm are presented in [6] and in the Appendix of the present paper.

\section{NUMERICAL RESULTS}

We consider fluid flow with particles through a pneumatic system that consists of two pipes with an initial larger diameter section, connected to a pipe with a smaller diameter (Figure 1). Initially particles with concentration $c$ are placed only in the large-diameter section, upstream from the small pipe. The physical parameters of the fluid and particles that were used in the numerical configurations are given in Table 1 . The boundary conditions consist of the inflow boundary conditions with the air velocity $u_{0}$ and zero Neumann boundary conditions for pressure at the left boundary and outflow boundary conditions and zero Dirichlet boundary conditions for pressure at the right boundary. We performed 16 numerical runs where we changed particle size, particle concentration, orifice diameters and air velocity (Table 2).

Figure 2 shows snapshot from a 3D simulation of solid particles conveying through the system (case R1). The fluid velocity in the smaller pipe reaches a local value of $200 \mathrm{~m} / \mathrm{s}$ with an averaged value in a cross section equal to $100 \mathrm{~m} / \mathrm{s}$. The flow behavior in the orifice region is quite complicated due to flow separation effects. Since the flow in constriction/expansion sections cannot follow the area change as suddenly as the geometry does, it results in flow separation with stagnation regions. Figure 2 shows flow stagnation regions by the white line. The particles tend to concentrate in the region of low shear in the middle section of the small diameter pipe immediately after entering the pipe.

Since we can calculate numerically $u_{g x}$ and $u_{s x}$ we can integrate (15) and find a semianalytical pressure distribution based on the computed fluid and solid velocities and particle concentrations in each cross section. This semi-analytical prediction is compared with the pressure computed directly from the simulations. The results of this comparison are presented in Figure 3. It's clearly seen in both approaches that the major pressure drop in the system occurs in the orifice area (in the transition area from the wide region to the narrow region). 
Pressure losses in the small pipe region are relatively small compared to this large pressure decrease at the entrance. Figure 3 shows that semi-analytical prediction underestimates the pressure in comparison to the simulations. The lower pressures indicated by the semianalytical model (15) are primarily because that model does not include additional losses due to flow separation and turbulent effects which are included in simulations. The initial pressures differ (i.e. in the large diameter pipe) because the semi-analytic expression value for the pressure in that region represents the integrated pressure decreases through the flow (scaled to have zero pressure at the exit point). Since fewer pressure loss mechanisms are included in (15), the apparent initial pressure appears as a lower value. However both numerical and theoretical approaches show that the major contribution to the pressure drop is due to slip in velocity between gaseous and solid phases which is included in both models and reaches maximum value in the orifice region. Acceleration of both gaseous and solid phases through the sudden-confinement causes a slip in velocity between them because the gaseous phase accelerates much faster than the solids, so the solid particles have a lower velocity than the gaseous phase in the confinement region. Since particles accelerate along the length of the pipe their velocity is different in each cross-section (Figure 4a). However because the solid flow rate is the same in each cross-section, the particle concentration decreases downstream (Figure 4b). This contributes to the overall pressure drop's increase which is proportional to $\frac{1}{(1-\varepsilon)}$ in (17). It is a known fact that for long pipes wall friction losses become a major contributor to the overall pressure drop. However, one can see that for the short and intermediate pipes considered here, the major pressure drop contributions are due to particle and flow acceleration effects.

To analyze particle collision effects we compare the averaged particle velocity for the particle concentrations of $c=0.01$ and $c=0.03$. Figure $4 \mathrm{a}$ shows that the averaged particle velocity is almost the same in both cases. Therefore we conclude that collision effects are negligible compared to the fluid-particle interacting effects for such low particle concentrations.

Then we considered the effects of pipe diameter and air velocity on the solid mass flow rate and pressure drop in the system. Figure 5 and Figure 6 show similar flow behavior that is characterized by the flow separation near the orifice region and particle concentration in the central regions of the pipe with further dispersion downstream from the orifice. Figure 7 shows the ratio of the solid velocity to the fluid velocity $\frac{u_{s}}{u_{f}}$ for particle sizes of 90 microns (Figure 7a) and 180 microns (Figure $7 \mathrm{~b}$ ). This ratio appears to be almost identical for the cases with the same particle sizes for different air flow conditions and orifice diameters and reaches the value of 1 for 90 micron particles and the value of 0.5 for 180 micron particles at the pipe exit. Therefore the $1.5 \mathrm{~cm}$ length of the pipe is sufficient for 90 micron particles to reach the air velocities at the pipe exit and 180 micron particles reach only half of the air velocity at the same distance from the orifice. 
Finally we compare pressure losses obtained numerically with pressure losses obtained by the derived analytical model (17) and find appropriate values of the discharge coefficient $K_{s}$. We extracted from the simulated pressure losses before the entrance and after the entrance in the small pipe and plot them against and the solid flow rates by different color dots in the diagram in Figure 9. It is clearly seen in Figure 9 that all these points lie on the same curve. To estimate the slope of this curve we computed pressure losses using equation (17) and computed solid mass flow rates at the exit of the small pipe. We found that for $K_{s}=10.98$ (or $k_{s}=0.61$ in (18)) theoretical data are very close to the numerical results (Figure 9). It is concluded that the derived expression (17) with $K_{s}=10.98$ describes pressure drop well in all scenarios considered in the present work for different particle sizes, particle concentrations, orifice sizes and air velocities.

\section{COMPARISON WITH EXPERIMENTAL DATA}

A set of laboratory experiments were conducted at LLNL which provide data for evaluation of the pneumatic system behavior. The flow chamber in these tests consisted of spherical container of diameter $15 \mathrm{~cm}$ connected with two small pipes: the inlet pipe of diameter $1 \mathrm{~mm}$ and length $1.5 \mathrm{~cm}$ and the outlet pipe of diameter $3 \mathrm{~mm}$ and length $3 \mathrm{~cm}$ (Figure 10). Approximately constant air flow with flow rate $42 \mathrm{~L} / \mathrm{min}$ was delivered to the container from an exterior system through the inlet pipe. The container was initially partially filled with spherical glass-bubble particles of diameter 45 microns and density $350 \mathrm{~kg} / \mathrm{m}^{3}$. Most of the pressure drop occurs in the inlet pipe, except when the mass flow of solids in the exit gets large and slows the overall gas flow. In that case a larger fraction of the total pressure drop in the system shifts to the exit pipe. The pressure drop in the outlet pipe was measured at approximately $\Delta P_{\text {air }}=20 \mathrm{kPa}$ for the air flow regime without particles. The total pressure drop in the outlet region and solid mass fluxes were measured during emptying phase of the container. It was found that the solid mass fluxes ranges from 0 to $7 \mathrm{~g} / \mathrm{s}$ and solid pressure $\left(\Delta P-\Delta P_{\text {air }}\right)$ ranges from 0 to $65 \mathrm{kPa}$ (Figure 12).

Since it is very computationally expensive to simulate the whole chamber with the current numerical approach we choose to simulate the small region near the outlet area which is shown by the red line in Figure 6. First we computed the air flow regime (without particles) and found that the pressure losses in the small outlet pipe with flow rate $42 \mathrm{~L} / \mathrm{s}$ was $\Delta P_{\text {air }}=18$ $\mathrm{kPa}$. This pressure drop in the short outlet pipe section we are modeling is slightly lower than $20 \mathrm{kPa}$ measured in the experiment due to additional pressure losses in downstream equipment (like pipe bends and the cyclone separator). We simulated flows with particles of concentration $\mathrm{c}=0.01, \mathrm{c}=0.03$ and $\mathrm{c}=0.05$. Figure 11 shows that there are large fluctuations on the sub-millisec timescale in the calculated solid mass fluxes. Since the experimental data points were averaged over quite long times (hundreds of ms per point), we did averaging over the sub-millisec fluctuations of the simulations to make it much easier to compare with the experimental data. Results of comparisons are shown in Figure 12. Both results were normalized on the same air pressure value of $\Delta P_{\text {air }}=20 \mathrm{kPa}$. One can see that slopes of experimental curve and simulations are very close to each other, though experimental data are somewhat scattered for high mass flow rates. Simulations with $1 \%, 3 \%$ and $5 \%$ of solids agree 
quite well with experimental data. We also did simulation with $10 \%$ of solids but the pressure drop in those simulations exceeded significantly the experimental range of pressure drops. Since the experimental data is the late time data, the concentration of $10 \%$ solids was probably not achieved at the final empting stage and it was always lower than that in experiment. Figure 12 also shows theoretical fit using expression (17) for $K_{s}=11.4$ (or $k_{s}=0.57$ in (18)).

A reasonably good agreement between theoretical, numerical and experimental results shown in Figure 12 confirms once again the consistency of numerical simulations and proposed approach to estimate pressure losses for low concentration pneumatic transport configurations with variable cross-sections.

\section{CONCLUSIONS}

Pressure and energy losses in the pipes with a step decrease in cross-sectional area are investigated numerically. We study correlations between pressure losses and solid mass fluxes in short pipes for different particle concentrations and particle sizes. Theoretical expressions that describe these correlations are derived and validated in several scenarios of particulate flows. We confirm that the major pressure losses in short pipes are attributed to the particle and flow acceleration effects in the system. The results of simulations agree reasonably well with experimental data. It is believed that the agreement between simulations and experimental data demonstrate the applicability of the proposed approach for prediction of the pressure losses in low concentration pneumatic transport in pipes with varying cross-sectional area.

\section{APPENDIX}

The governing equations and algorithm are presented below. The particle domain is denoted as $P(t)$, where $\partial \mathrm{P}$ is the interface between the particle and the fluid. $F$ is the fluid domain that is not shared with the particle. The entire computational domain that includes both the fluid and the particles is denoted by $F \cup P$. The governing equations in the fluid domain can be written as:

$$
\begin{aligned}
& \rho\left(\frac{\partial \vec{u}}{\partial t}+(\vec{u} \cdot \nabla) \vec{u}\right)=\nabla \cdot \sigma+\rho g, \text { in } F \cup P \\
& \nabla \cdot \vec{u}=0, \text { in } F \cup P \\
& D[\vec{u}]=0, \text { in } P(t) \\
& D[\vec{u}] \cdot \vec{n}=0, \text { on } \partial P(t)
\end{aligned}
$$




$$
\vec{u}=\vec{u}_{s}, \text { in } P(t)
$$

where $\vec{u}$ is the fluid velocity, $p$ is the pressure, $\rho$ is the density that is equal to $\rho_{f}$ in the fluid domain and equal to $\rho_{s}$ in the particle domain, $g$ is gravity, $\vec{n}$ is a unit normal on the particle surface. It should be noted that boundary condition (23) precludes penetration of fluid into the grain. If $\vec{u}_{s}=0$ (23) describes the slip condition $\vec{u}=0$ for fluid.

The rigid body velocity inside the particle us is represented as

$$
\vec{u}_{s}=\vec{U}+\vec{\Omega} \times \vec{r}
$$

where $\vec{U}$ and $\vec{\Omega}$ are the translational and angular velocities of the particle, respectively, and $\vec{r}$ is the position vector of the point with respect to the particle centroid. $D[\vec{u}]$ is the deformation-rate tensor defined as

$$
D[\vec{u}]=\frac{1}{2}\left[\nabla \vec{u}+\nabla \vec{u}^{T}\right]
$$

The stress tensor in the fluid domain is then given by:

$$
\sigma=-p I+\tau
$$

where $I$ is the identity tensor, $p$ is the pressure and $\tau$ is the viscous stress tensor given by:

$$
\tau=\mu D[\vec{u}]
$$

for a Newtonian fluid, where $\mu$ is the viscosity of the fluid. The particle domain in this formulation is treated as a fluid with an additional constraint (Lagrange multiplier) to impose the rigid body motions in such a way that deformation-rate tensor $D[\vec{u}]$ within the particle domain is zero. The stresses inside the particle [10] is given by

$$
=p I++D[]
$$

The integration of governing equations is done using a fractional time stepping approach at time interval $\left[\mathrm{t}^{\mathrm{n}}, \mathrm{t}^{\mathrm{n}+1}\right]$. In the present algorithm, the velocity and pressure are cell-centered quantities. The velocity is defined at integer multiples of $\Delta t$, whereas the pressure is defined at half-time steps. The system of equations (19)-(21)- with boundary conditions (22)-(24) are solved numerically using the operator-splitting technique that combines several steps described below. 
First step: We solve the Navier-Stokes equations (19), (20) on the Eulerian grid. We neglect the presence of the particles at this point, except for their density. Since the particle size is greater than cell size, we project the particles onto the Eulerian fluid grid by computing a volume fraction of each particle $\phi$, which ranges between 0 if the grid cell is completely outside any particles, and 1 if it completely inside. An unsplit second order Godunov procedure is used to approximate the non-linear advection term that appears in the momentum equations using both velocities defined at the centers of the Cartesian grid as well as velocities defined at the cell faces. The density is computed as $\rho=\rho_{s} \phi+\rho_{f}(1-\phi)$, where $\rho_{s}$ is the particle density and $\rho_{f}$ is the fluid density, which are constants determined from the material properties of the fluid and grains. Velocity field $\overrightarrow{\tilde{u}}$ is found at this time step.

Second step: The code uses a Lagrange multiplier method to enforce the correct no-slip boundary condition over the surface of the particles and inside them. We enforce rigid body motion on the particles by taking the average translational and rotational velocities within a small region within a single grain, and setting it equal to the particle's Lagrangian velocity. The velocity at each Eulerian grid point is updated accordingly. The particle velocity in a given cell is split into translational $\vec{U}$ and rotational $\vec{\Omega}$ parts as

$$
\vec{u}_{s}=\vec{U}+\vec{\Omega} \times \vec{r}
$$

where $\vec{r}$ is a vector which connects a particle centroid and a center of the considered grid cell. Particle velocities $\vec{U}$ and $\vec{\Omega}$ are computed by integration of the provisional velocity field $\overrightarrow{\widetilde{u}}$ in the solid domain as

$$
\begin{aligned}
& M \vec{U}=\int_{P} \rho_{s} \tilde{u} d V \\
& I_{p} \vec{\Omega}=\int_{P} r \times \rho_{s} \tilde{u} d V
\end{aligned}
$$

where $M$ is the mass of a particle and $I_{p}$ is the moment of inertia of a particle. In simulations in this paper we used spherical particles so the moment of inertia is a scalar constant. Once they found the particle velocity is computed from (29). The velocity field $\vec{u}^{n+1}$ is then updated in the solid, fluid and mixed domains (where both solid and fluid are presented) as 


$$
\begin{cases}\vec{u}^{n+1}=\vec{u}_{s}, & \text { in solid cells } \\ \vec{u}^{n+1}=\overrightarrow{\tilde{u}}, & \text { in fluid cells } \\ \vec{u}^{n+1}=\frac{\rho_{s} \varphi \vec{u}_{s}+\rho_{f}(1-\varphi) \overrightarrow{\tilde{u}}}{\rho}, & \text { in mixed cells }\end{cases}
$$

where $\phi$ is the solid volume of fraction, computed based on the particle position at time $n$. This step could be considered as finding force $\vec{f}$ that modifies the provisional velocity field $\overrightarrow{\tilde{u}}$ such that the final velocity field $\vec{u}^{n+1}$ satisfies rigid body motion constraints.

Third step: If the particle Reynolds number and solid volume fraction are low, particles do not interact, and $U$ defined at the previous step are the final velocities that describe the velocity field inside the particle. When the concentration of particles is high enough, they begin to interact with each other. If the viscosity of the fluid is high it will prevent particles from colliding each other. Treatment of collisions includes both the contact detection algorithm and applying the collision forces to prevent particles from overlapping. In the present work the contact is detected in the region where two particles overlap based on the volume fraction function $\phi$ (Figure 13). The condition $\phi>1$ means that more than one particle exists in the given grid cell, therefore an additional collision force contributions is applied in each grid cell where particles overlap. As we resolve the particle domain on a stationary grid, we compute collision force in each cell of the overlapping region and then integrate them through the entire overlapping volume. The collision force acts along the normal ( $\vec{F}_{n}$-component) as well as the tangential direction ( $\vec{F}_{t}$-component) at the point of contact between two particles (Figure 13). For spherical particles, the normal and tangential directions are defined by the line joining the centers of two colliding particles and plane perpendicular to it, respectively (Figure 13). The components of the normal collisional force is computed in each cell where particles overlap and then integrated over the overlapped region as

$$
\vec{F}_{n}=-\sum_{i j k}\left(k_{n} V_{i j k}+d_{n} V_{i j k} v_{n}\right) / m_{p}
$$

where $V_{i j k}$ - volume of cell $i j k$ in the overlapped region, $v_{n}$ - relative velocity between the two interacting particles in the normal direction, $k_{n}$ - normal spring constant (or stiffness), $d_{n}$ - damping coefficient in the normal direction, $m_{p}$ - particle mass. It was shown in Walton, (1993) that including both velocity and displacement in the viscous damping term in (33) is efficient to eliminate a discontinuity in the force that arises in a linear damped oscillator model when initial contact is established. 
The tangential force component is proportional to the relative tangential velocity $\vec{v}_{t}$ up to the Coulomb friction

$$
\vec{F}_{t}= \begin{cases}\sum_{i j k} d_{t} V_{i j k} \vec{v}_{t}, & \left|F_{t}\right| \leq \mu_{f}|F n| \\ -\mu_{f}|F n| \vec{t}, & \left|F_{t}\right| \geq \mu_{f}|F n|\end{cases}
$$

where $\vec{v}_{t}$ - relative velocity between the two interacting particles in the tangential direction $\vec{t}$, $\mu_{f}$ is the friction coefficient, and $d_{t}$ is the damping coefficient in the tangential direction. Those values are related to the coefficient of restitution of the grains. A discussion of how damping and stiffness affect the numerical results can be found in [6]. For the numerical results presented in this paper the particle concentration was less than $10 \%$, therefore collision parameters did not affect significantly results.

It can be noticed that the collision force $\vec{F}^{n+1}$ applied to the grains is somewhat similar to DEM models (Cundall and Strack, 1979). In our case DEM forces are applied in each individual grid cell on the overlapping region and then integrated over this region to provide the net response (Figure 13). It should be noted that force $\vec{F}^{n+1}$ affects only velocity distribution inside the grain.

Geometrically, the collision force depends on the overlap volume between two particles. In the case of particle-wall collisions the computational procedure is trivially extended, taking into account that velocity of walls is zero. In the general case the stiffness and damping coefficients for particle-wall and particle-particle interactions could be different.

Fourth step: Once collision forces are calculated, the final particle velocities (translational and rotational) are updated using the velocity field from previous time step as:

$$
\begin{aligned}
& \vec{U}^{n+1}=\overrightarrow{\widetilde{U}}^{n+1}+\vec{F}^{n+1 / 2} \Delta t \\
& I_{p} \vec{\Omega}^{n+1}=I_{p} \overrightarrow{\tilde{\Omega}}^{n+1}+\left[\vec{r} \times \vec{F}^{n+1 / 2}\right] \Delta t
\end{aligned}
$$

where $\vec{U}^{n+1}$ and $\vec{\Omega}^{n+1}$ are particle velocities computed at the previous stage.

Finally, we update the Lagrangian position of each particle by the following procedure

$$
X^{n+1}=X^{n}+U^{n+1 / 2} t
$$

\section{Acknowledgments}

This work performed under the auspices of the US Department of Energy by Lawrence Livermore National Laboratory under Contract DE-AC52-07NA27344. We thank Brian Comaskey for providing experimental data. 


\section{References}

[1] Cundall P.A., and O. D. Strack: A discrete numerical model for granular assemblies, Geotechnique, 29 (1979) 47.

[2] Glowinski R., T. W. Pan, T.I. Hesla, D. D. Joseph. A distributed Lagrange multiplier fictitious domain method for particulate flow. Int. J. of Mult. Flow, 25 (1999), 755-794.

[3] Griffith B.E., R.D. Hornung, D.M. McQueen, and C.S. Peskin, 2007: An adaptive, formally second order accurate version of the immersed boundary method. J. Comp. Phys., 223, 10-49.

[4] Griffith B.E., R.D. Hornung, D.M. McQueen, and C.S. Peskin. Parallel and Adaptive Simulation of Cardiac Fluid Dynamics. In: Advanced Computational Infrastructures for Parallel and Distributed Adaptive Applications. M. Parashar, S. Chandra, and X. Li, eds. John Wiley and Sons.

[5] Gundogdu M. Y., A. I. Kutlar, H. Duz. Analytical prediction of pressure loss through a sudden-expansion in two-phase pneumatic conveying system. Advanced Powder technology, 20 (2009), 48-54.

[6] Kanarska Y., I. Lomov, T. Antoun: Mesoscale Simulations of Particulate Flows with Parallel Distributed Lagrange Multiplier Technique. Comp. and Fluids, 48(1) (2011), 1629.

[7] Kojasoy G., F. Landis, P. Kwame-Mensah, C.T. Chang. "Two-phase pressure drop in multiple thick- and thin-orifice plates". Experimetnal Thermal and Fluid Science, 15, 347358 (1997)

[8] Marcus R. D., L.S. Leung, G.E. Klinzing, F. Rizk: Pneumatic conveying of solids. A theoretical and practical approach. Third edition. Springer. (2010), 575.

[9] Martin J. J. and V.R. Pabbi. Use of momentum balance in calibrating orifices for flow of gases. A. I. Ch. E. Journal, 6(2), 318-321, 1960.

[10] Patankar N.A.: A formulation for fast computations of rigid particulate flows. Center Turbul. Res. Ann. Res. Briefs (2001), 185-196.

[11] Peskin, C.S. The immersed boundary method. Acta Num., 11, 479 (2002).

[12] Rhodes M. Pneumatic transport of powders. Educ. Reso. For Part. Techn. (2001). http://www.erpt.org/014Q/rhoe-00.htm

[13] Walton, O.R. "Chapter 25: Numerical Simulation of Inelastic Frictional ParticleParticle-Interactions," Particulate Two-Phase Flow M.C. Roco, ed., ButterworthHeinemann, Stoneham, MA, (1993) 
Table 1 Physical properties of particles and air

\begin{tabular}{|l|l|}
\hline Parameter & Value \\
\hline Particle density & $350 \mathrm{~kg} / \mathrm{m}^{3}$ \\
\hline Fluid density & $1 \mathrm{~kg} / \mathrm{m}^{3}$ \\
\hline Fluid viscosity & $2 \times 10^{-5} \mathrm{~Pa} \cdot \mathrm{s}$ \\
\hline Coefficient of friction & 0.3 \\
\hline Stiffness & $5000 \mathrm{~N} / \mathrm{m}^{3}$ \\
\hline Damping coefficient & 0.5 \\
\hline Particle shapes & Spherical \\
\hline Large pipe diameter & $15 \mathrm{~mm}$ \\
\hline Small pipe length & $15 \mathrm{~mm}$ \\
\hline
\end{tabular}

Table 2 Parameters for different numerical runs

\begin{tabular}{|l|c|c|c|c|}
\hline Case & $\begin{array}{c}\text { Particle diameter, } \\
\text { microns }\end{array}$ & $\begin{array}{c}\text { Small pipe } \\
\text { diameter, } \mathrm{mm}\end{array}$ & $\begin{array}{c}\text { Initial velocity, } \\
\mathrm{m} / \mathrm{s}\end{array}$ & $\begin{array}{c}\text { Initial particle } \\
\text { concentration, } \%\end{array}$ \\
\hline R1 & 180 & 3 & 100 & 1 \\
\hline R2 & 180 & 3 & 100 & 3 \\
\hline R3 & 90 & 3 & 100 & 1 \\
\hline R4 & 90 & 3 & 100 & 3 \\
\hline R5 & 180 & 3 & 220 & 3 \\
\hline R6 & 180 & 3 & 220 & 1 \\
\hline R7 & 90 & 3 & 220 & 3 \\
\hline R8 & 90 & 3 & 220 & 3 \\
\hline R9 & 180 & 2 & 100 & 3 \\
\hline R10 & 180 & 2 & 220 & 3 \\
\hline R11 & 90 & 2 & 100 & 3 \\
\hline R12 & 90 & 2 & 220 & 3 \\
\hline R13 & 180 & 4 & 100 & 3 \\
\hline R14 & 180 & 4 & 220 & 3 \\
\hline R15 & 90 & 4 & 100 & 220 \\
\hline R16 & 90 & 4 & & \\
\hline
\end{tabular}




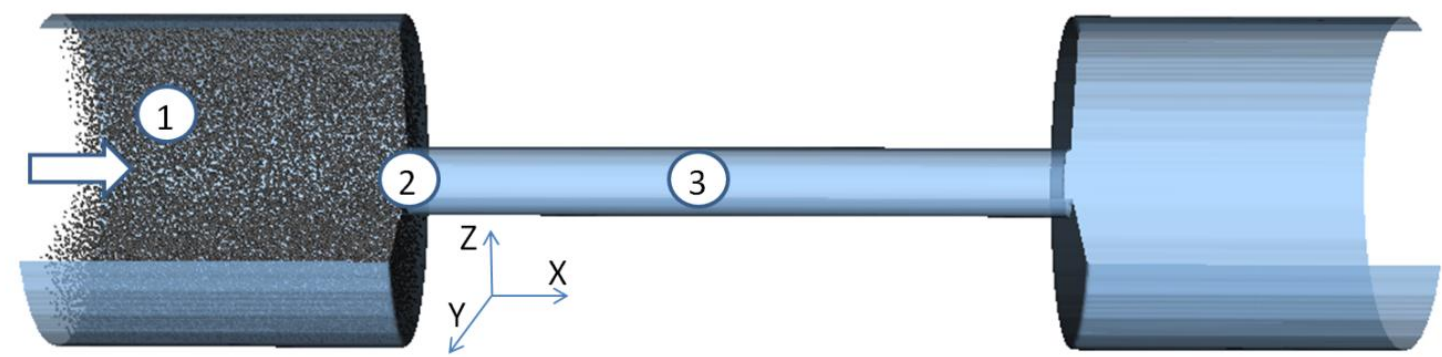

Figure 1. Schematic description of the pneumatic system with variable cross-sectional area.

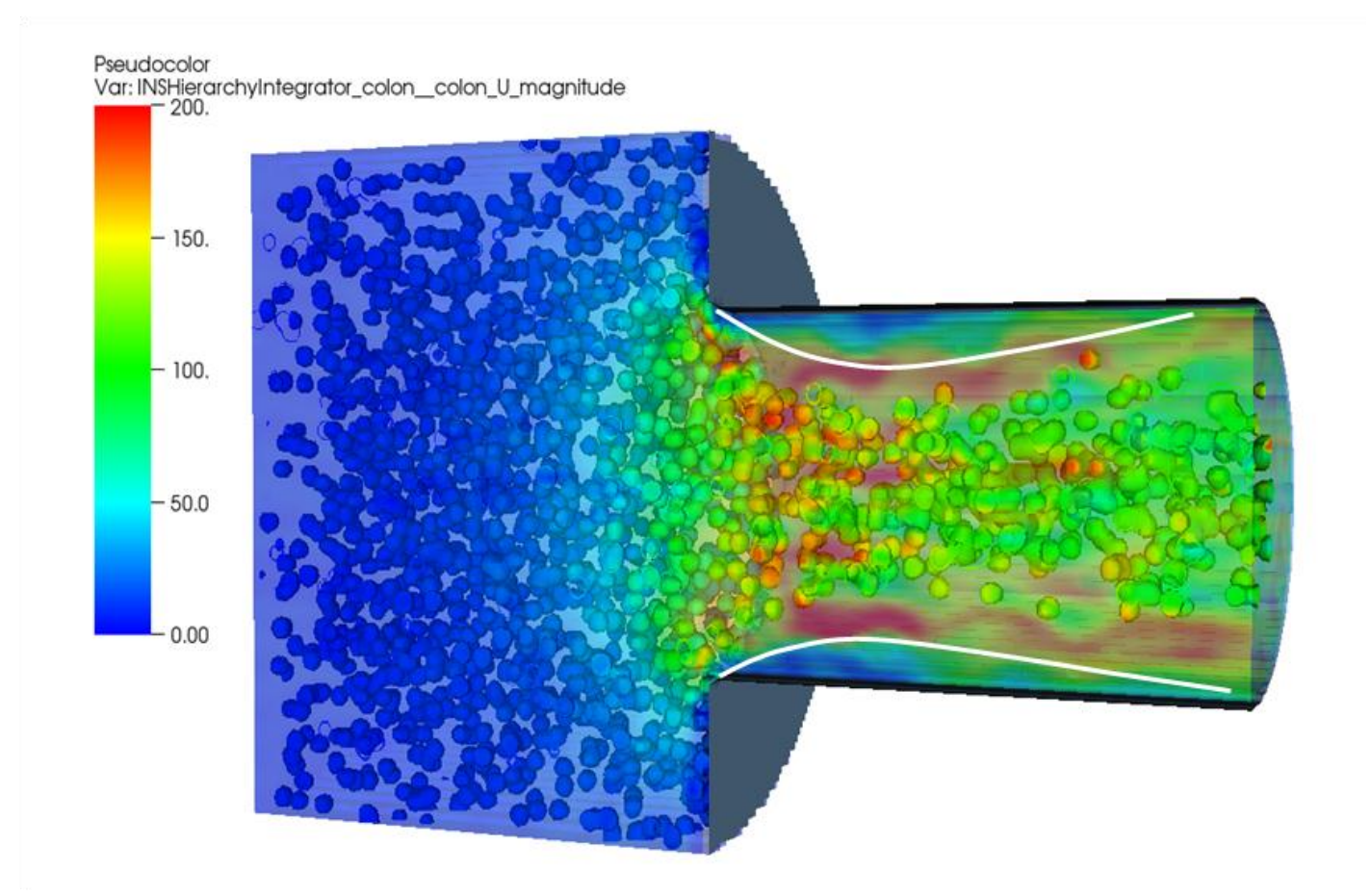

Figure 2. Particle conveying through the pipe for case R1 (zoom of simulation in the orifice area). Fluid velocity field is shown by color. The white line shows flow separation and stagnation regions. 


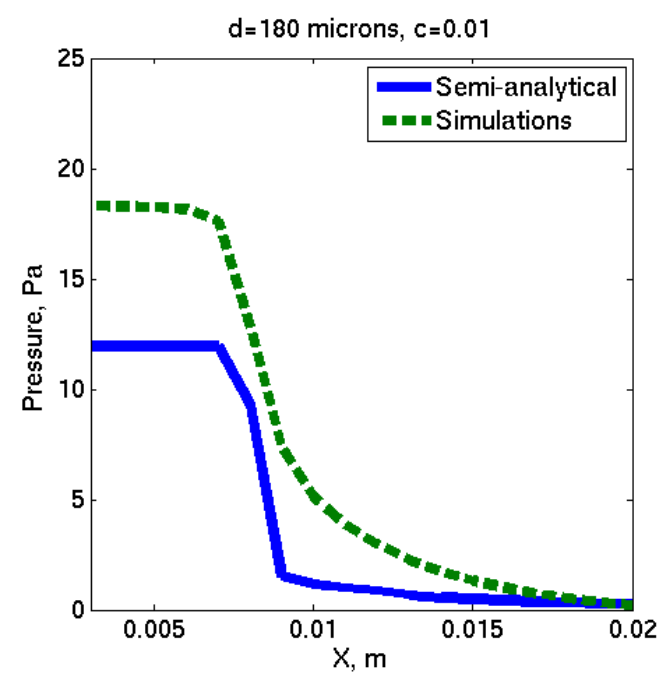

(a) case R1: size 180 micron, $c=1 \%$

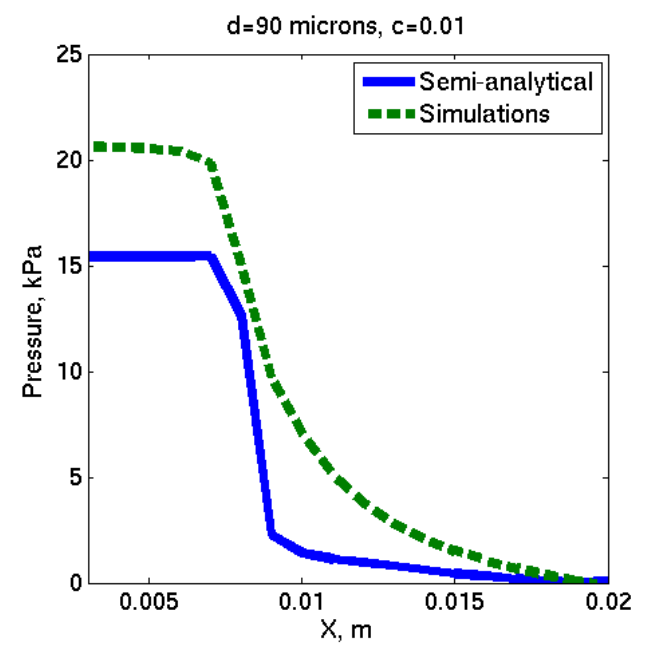

(c) case R3: size 90 micron, $c=1 \%$

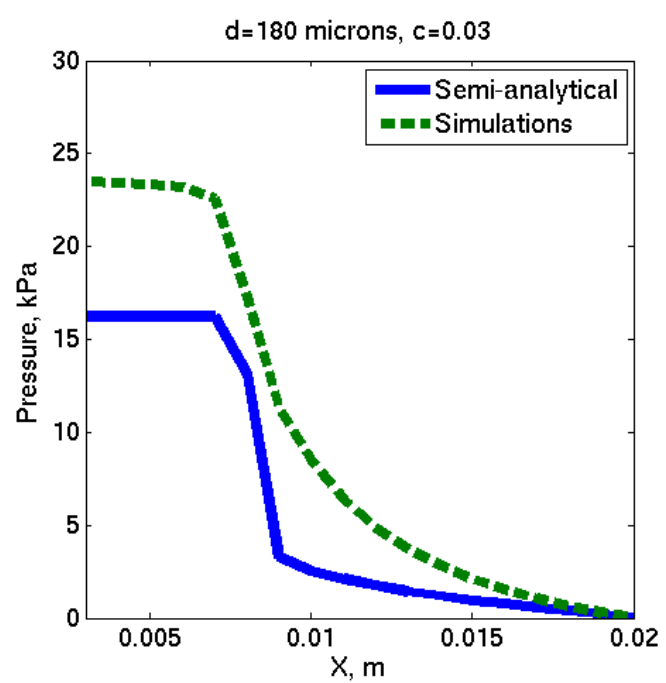

(b) case R2: size 180 micron, $c=3 \%$

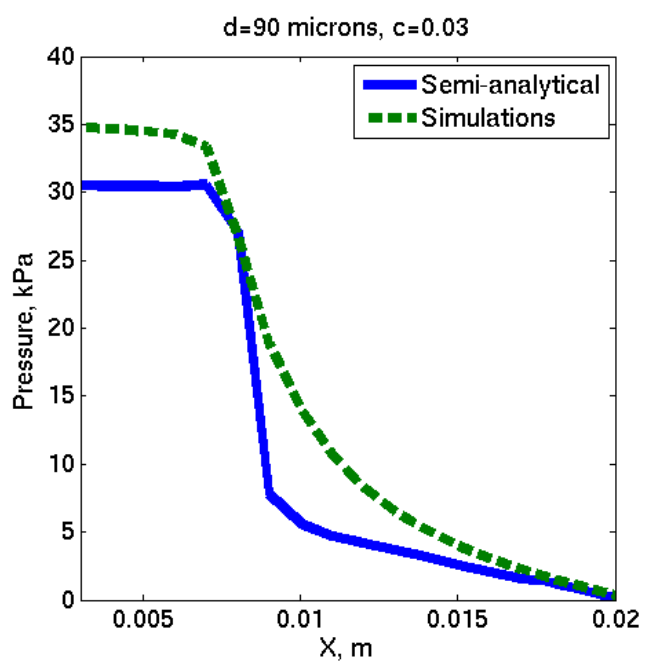

(d) case R4: size 90 micron, $c=3 \%$

Figure 3. Pressure drop along X-axis computed directly from simulations and derived from equation (14) by neglecting frictional forces 


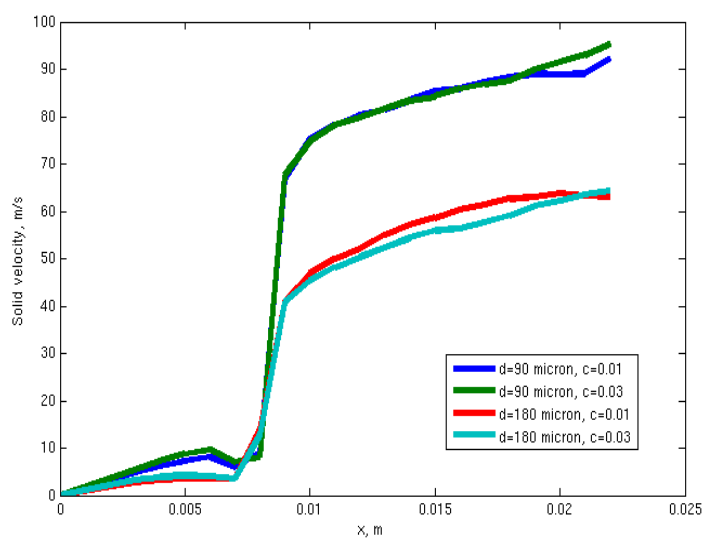

(a) Particle velocity

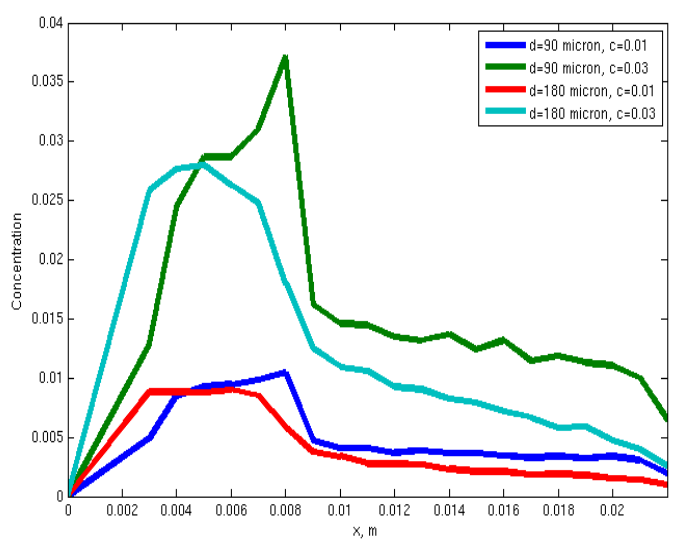

(b) Particle concentration

Figure 4. Averaged particle properties along X-axis. 

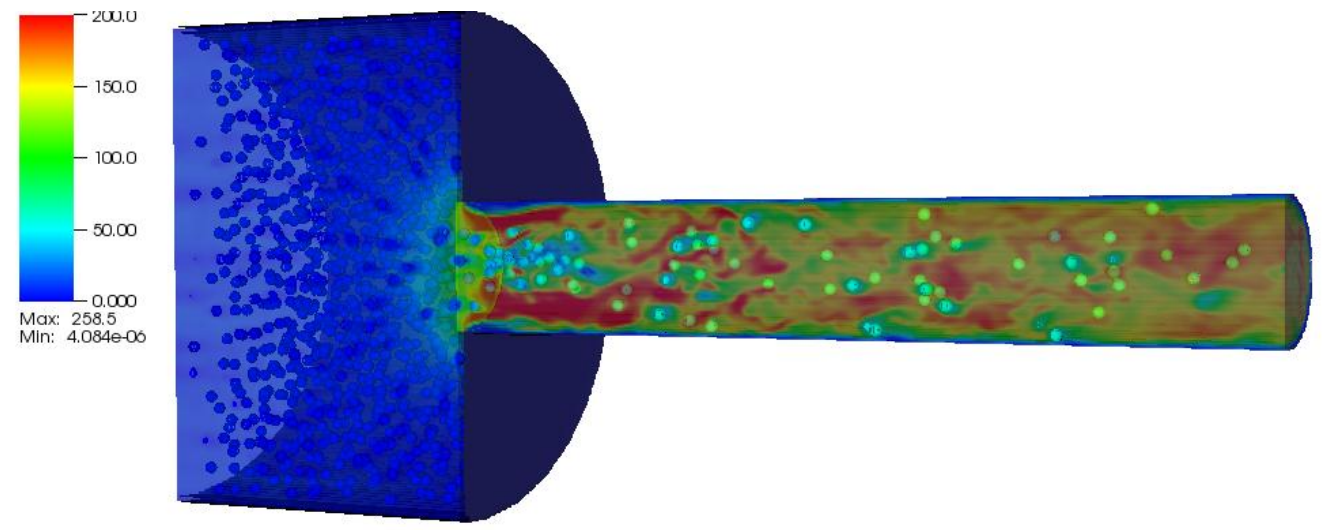

(a) pipe diameter is $2 \mathrm{~mm}$

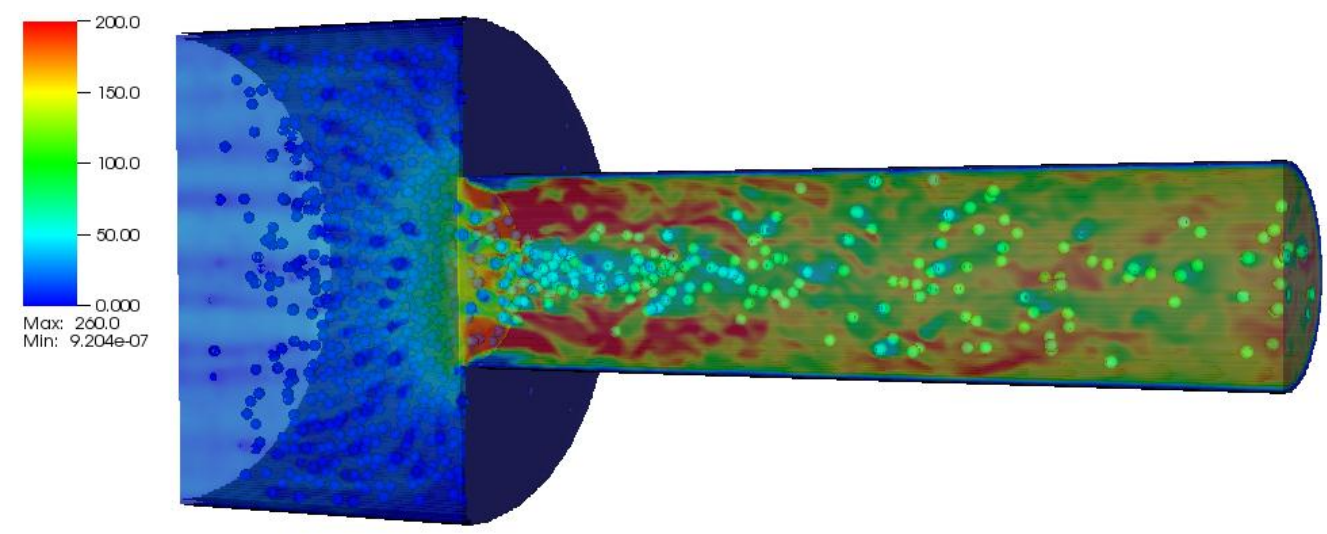

(b) pipe diameter is $3 \mathrm{~mm}$
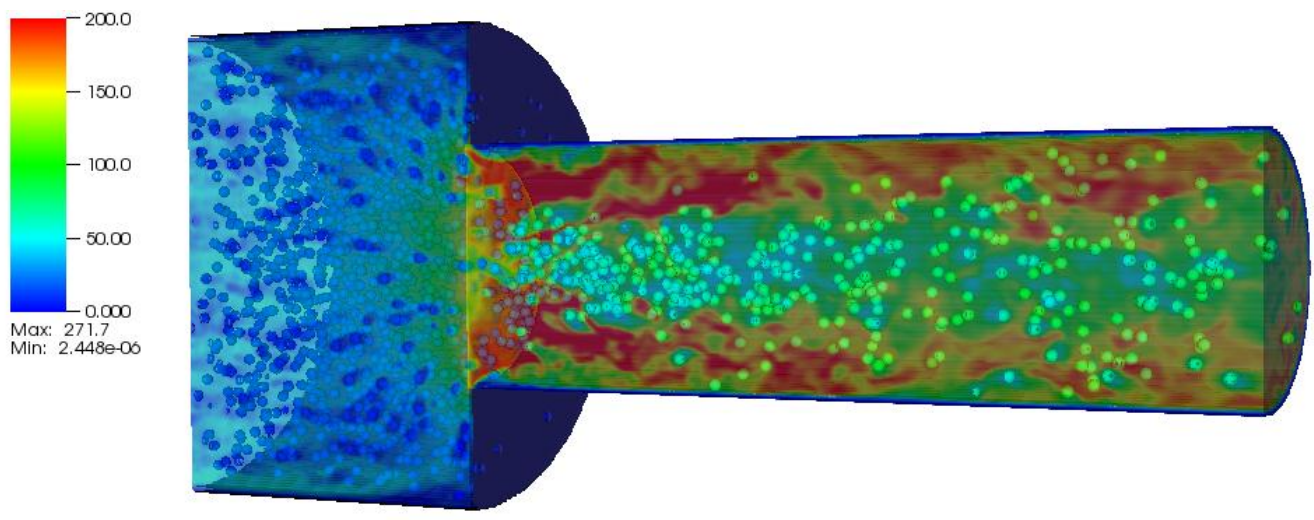

(c) pipe diameter is $4 \mathrm{~mm}$

Figure 5. Particle conveying through the pipes of different diameters. Initial particle concentration is 0.03 , particle diameter is 180 microns (cases R9, R2 and R13). Fluid velocity field is shown by color. 

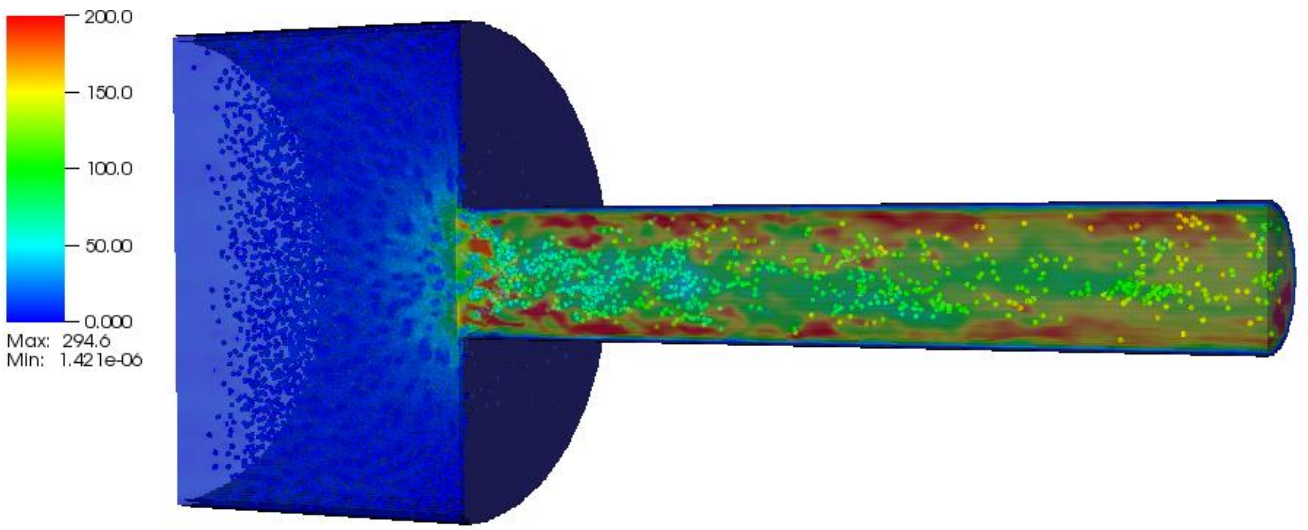

(a) pipe diameter is $2 \mathrm{~mm}$

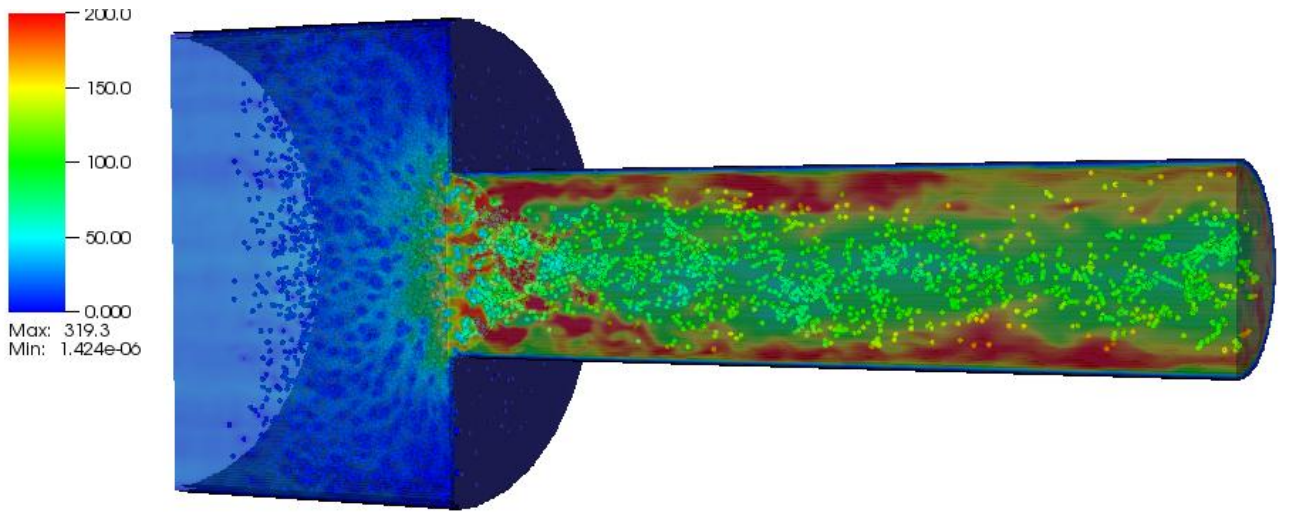

(b) pipe diameter is $3 \mathrm{~mm}$

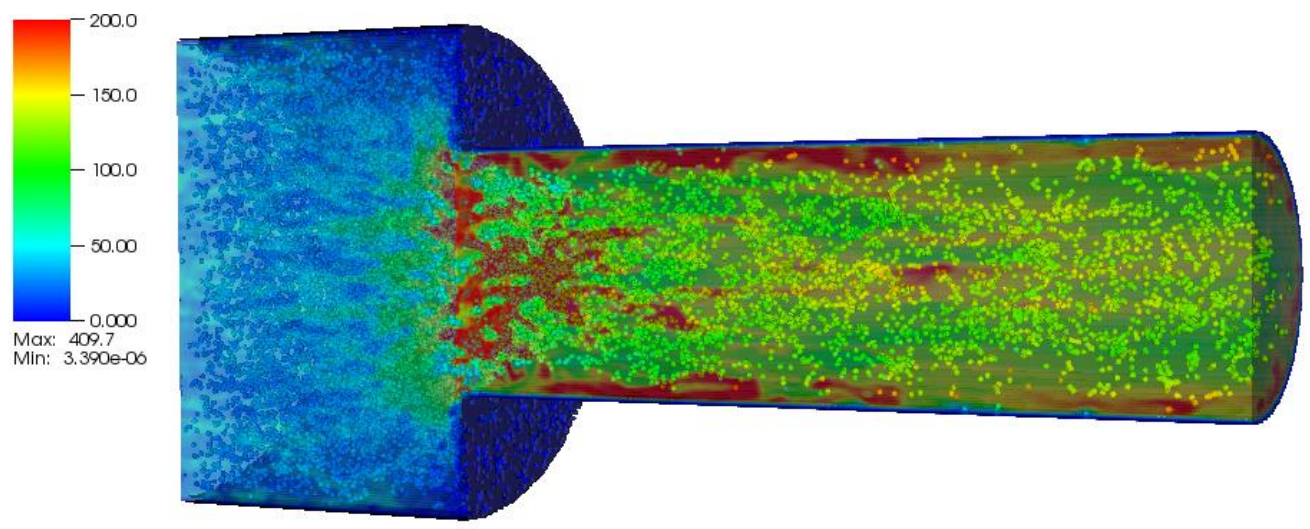

(c) pipe diameter is $4 \mathrm{~mm}$

Figure 6. Particle conveying through the pipes of different diameters. Initial particle concentration is 0.03 , particle diameter is 90 microns (cases R11, R4, R15). Fluid velocity field is shown by color. 


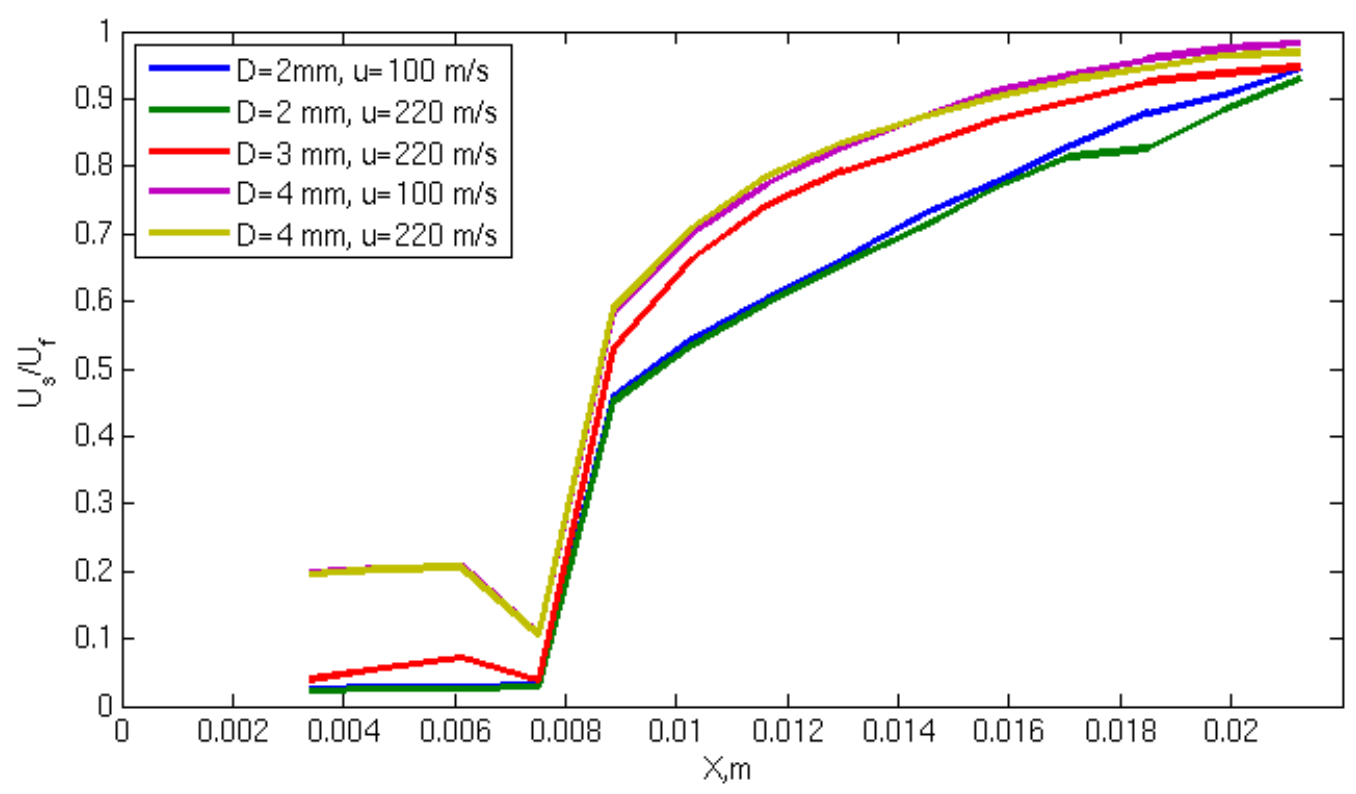

(a)

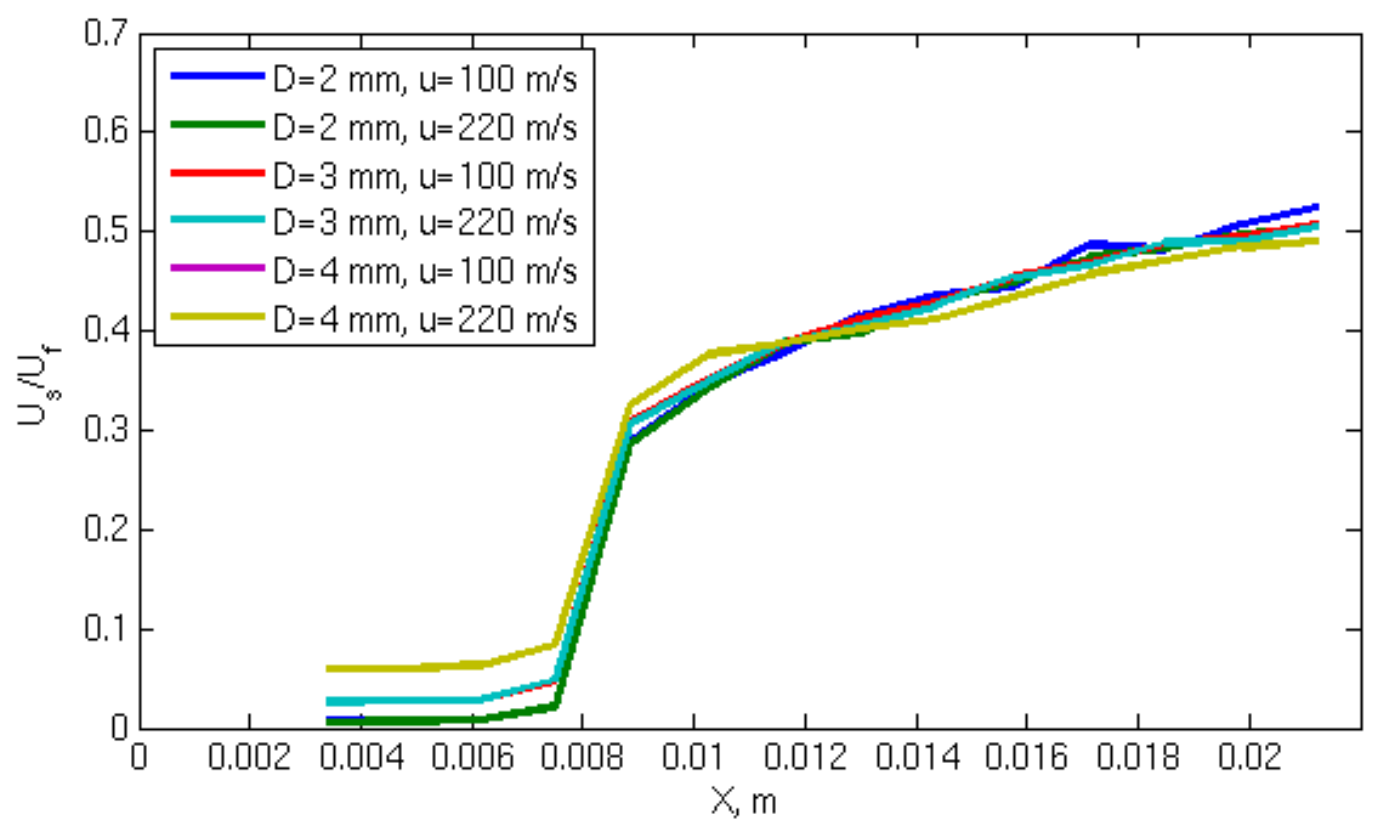

(b)

Figure 7. Relative velocity in the pipe. Initial particle concentration is 0.03 , particle diameter is 90 microns (a) and 180 microns (b). 


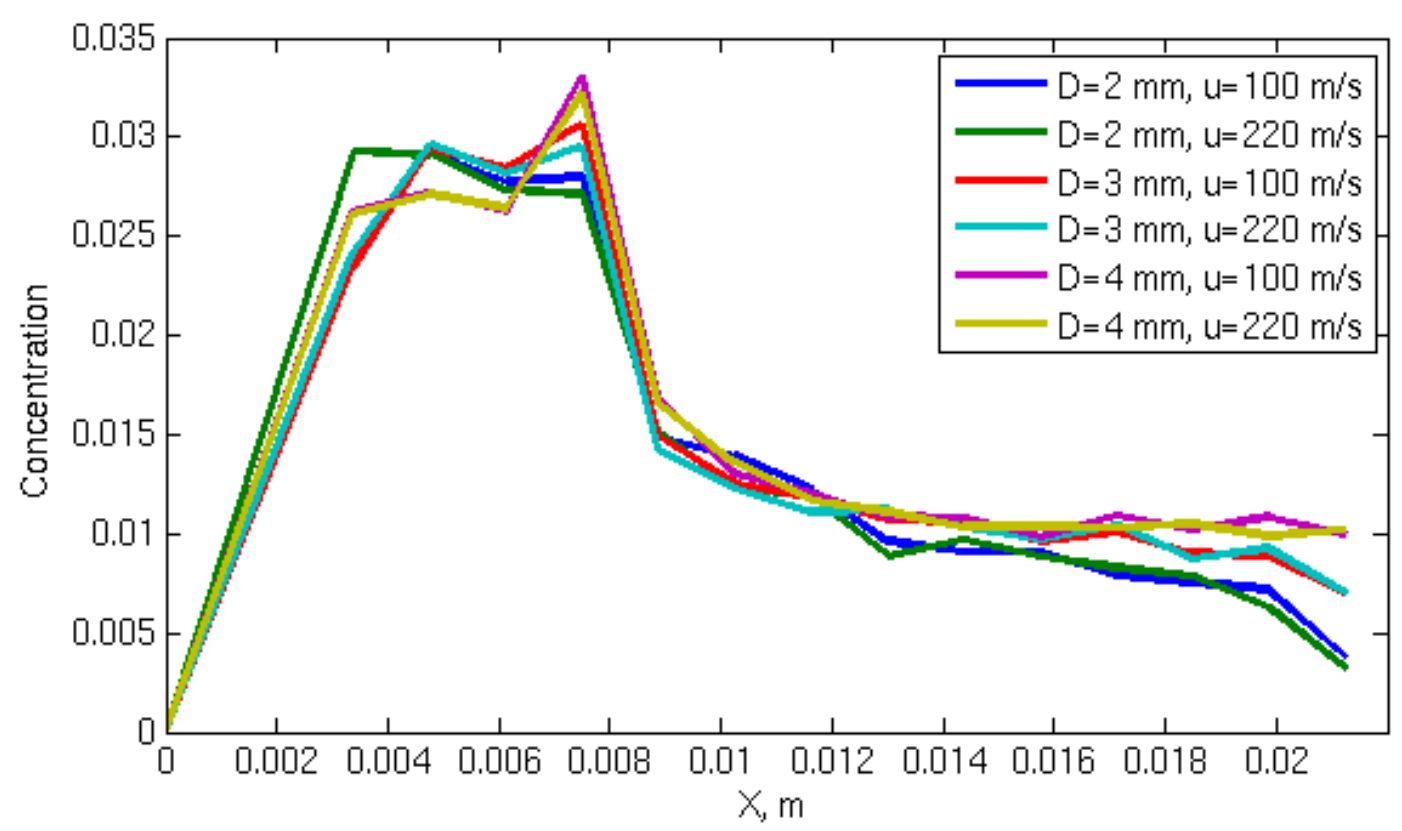

(a)

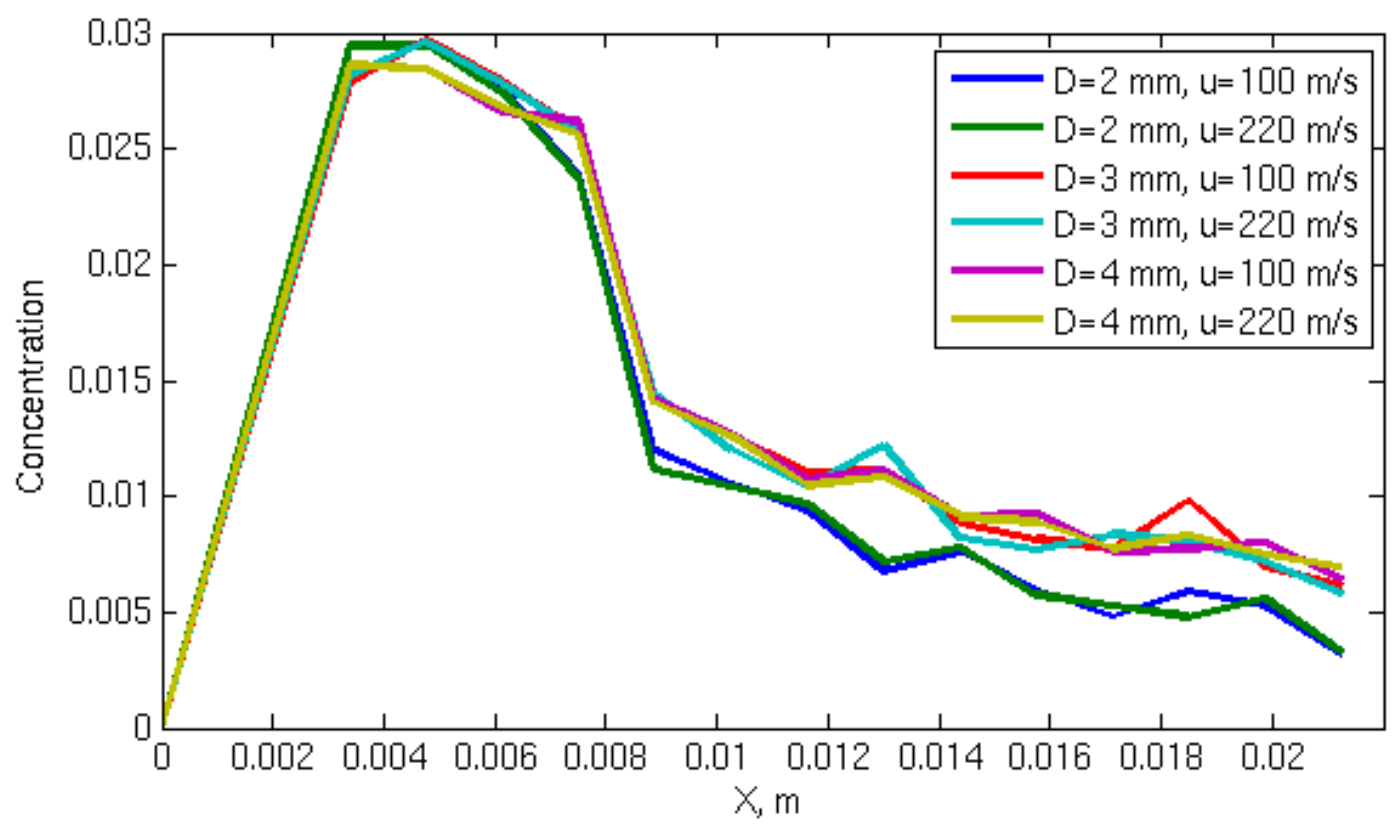

(b)

Figure 8. Particle concentration in the pipe. Initial particle concentration is 0.03 , particle diameter is 90 microns (a) and 180 microns (b). 


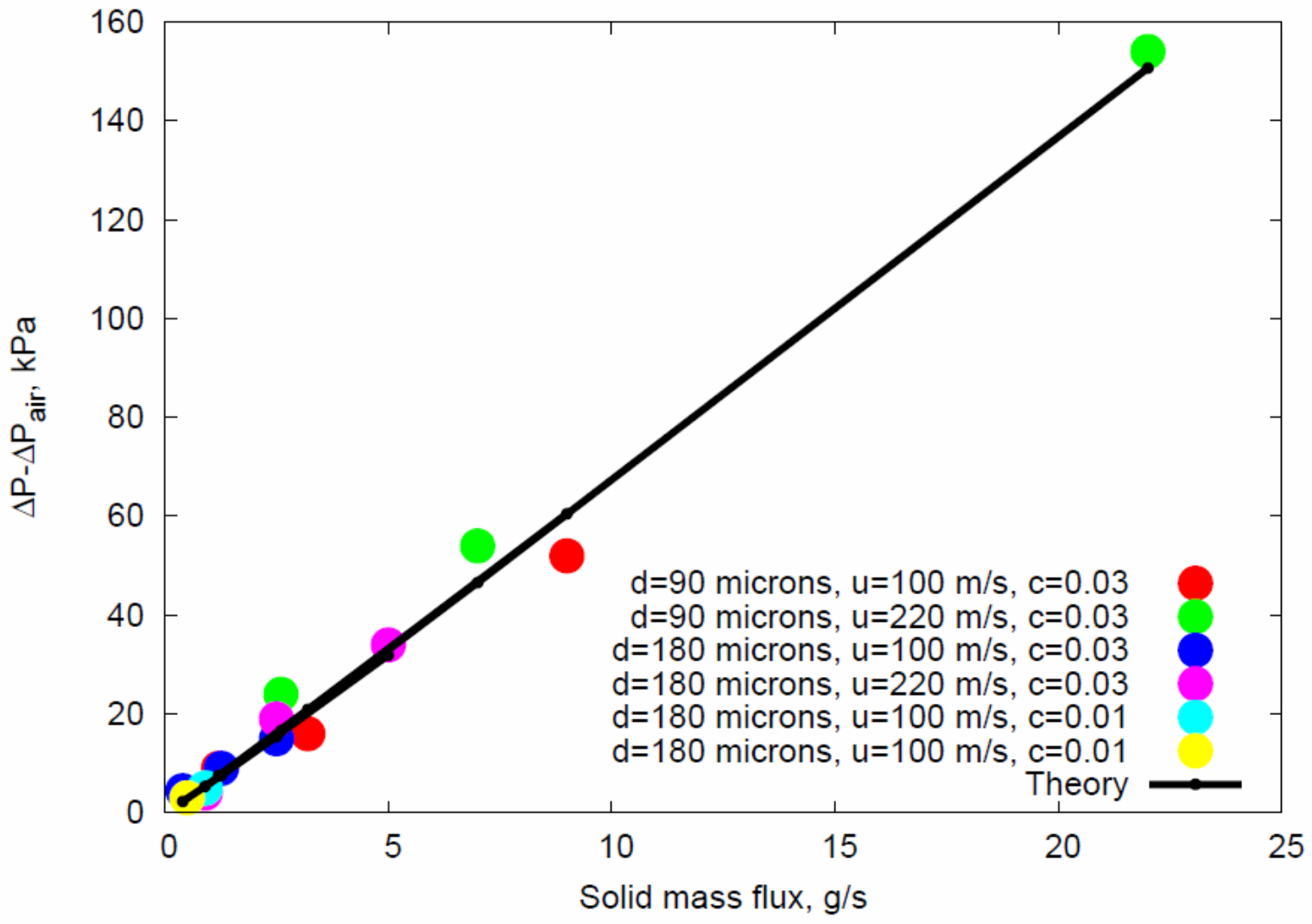

Figure 9. Pressure drop versus solid mass fluxes for all cases R1-R16 and theoretical fit using (17). 


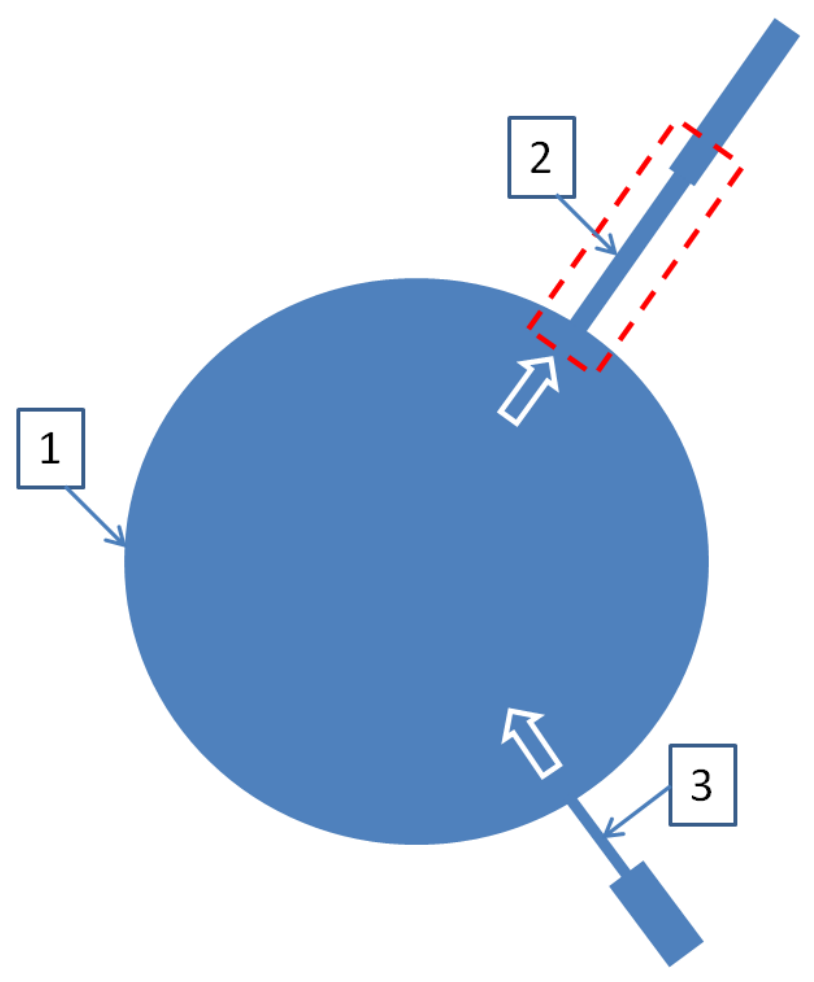

Figure 10. Schematic representation of the experimental setup. 1-spherical container, 2-outlet pipe, 3-inlet pipe. Red line shows simulated domain.

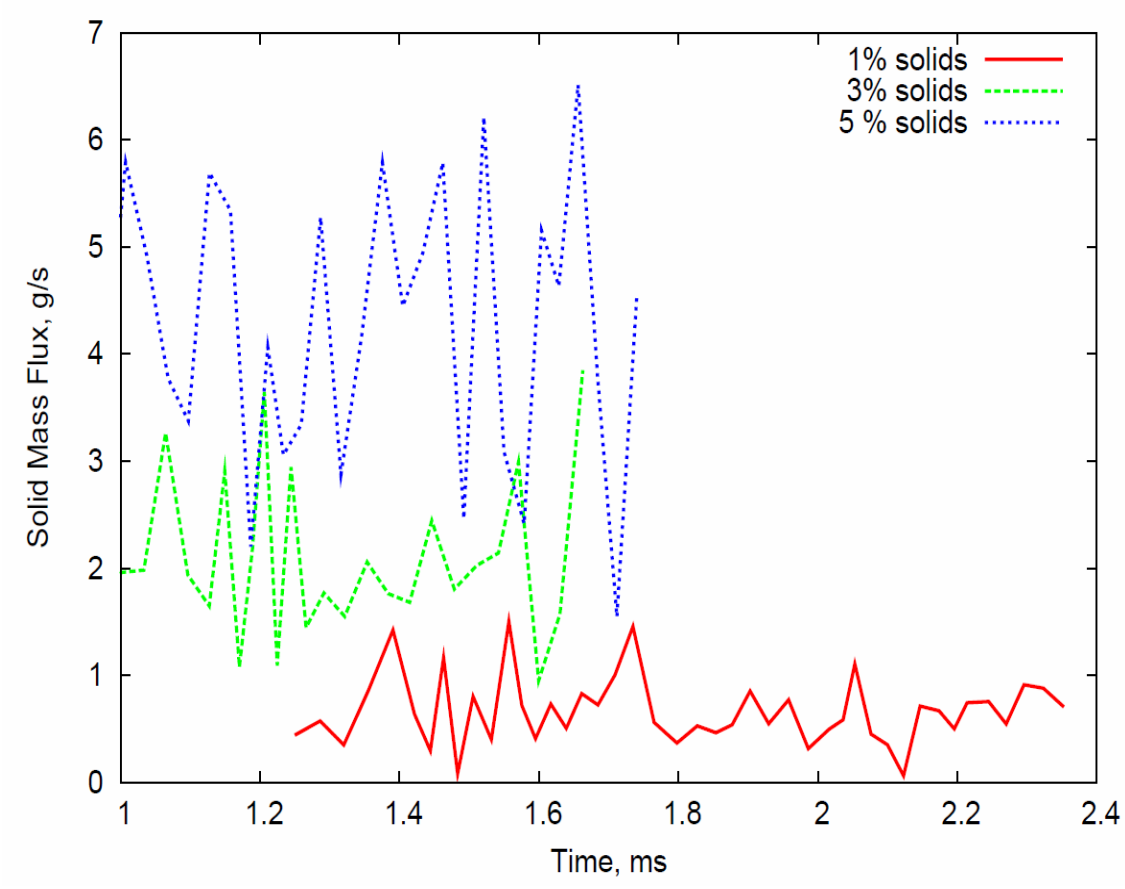

Figure 11 Solid mass flux fluctuations in simulations. 


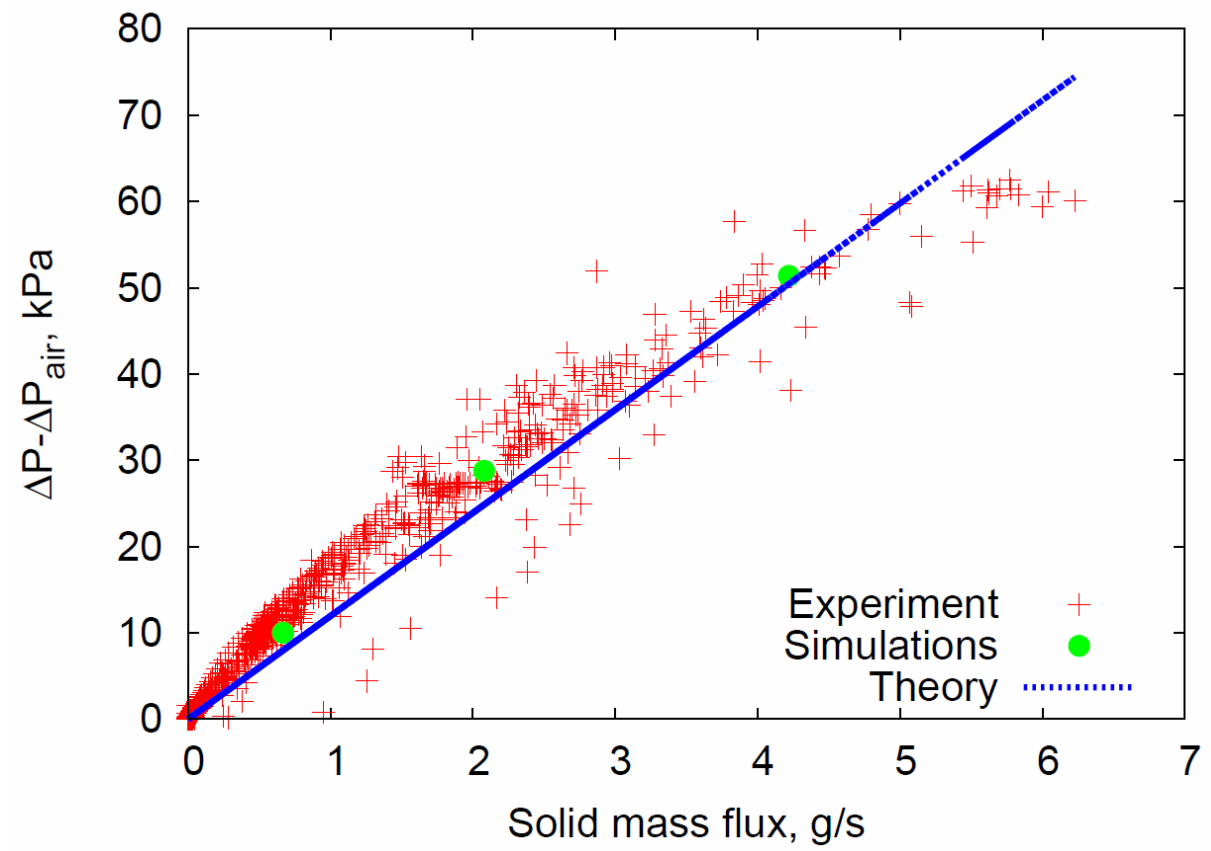

Figure 12. Pressure drop versus dimensionless pressure in experiment and simulations.

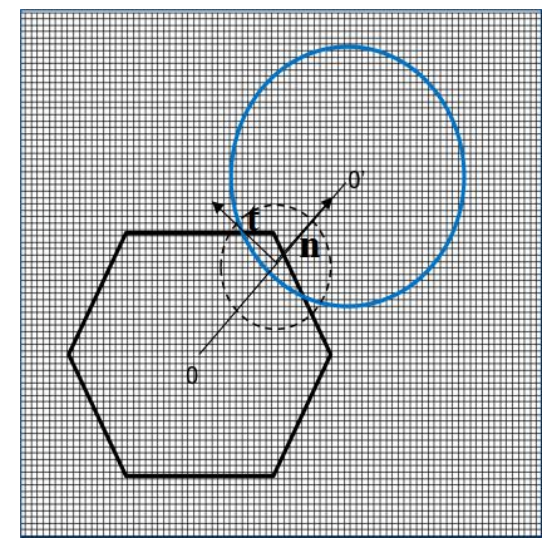

Figure 13 Schematic description of particle interaction. Collision forces are applied in the normal and tangential direction and computed in the overlapping region that consists of several grid points. 


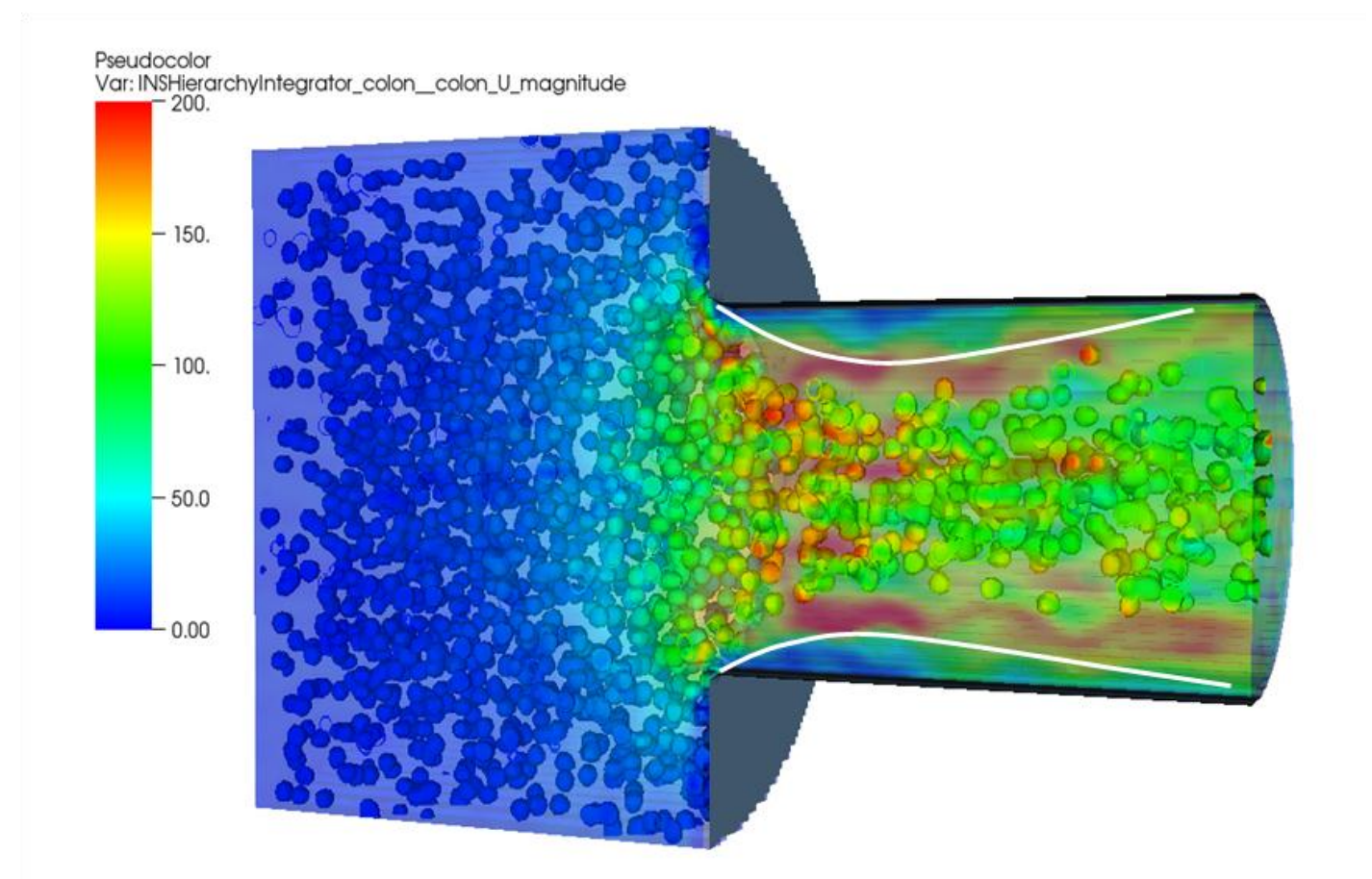

Particle conveying of 180 micron particles through the orifice of diameter $3 \mathrm{~mm}$. Fluid velocity field is shown by color. The white line shows flow separation and stagnation regions. 